\title{
MOLECULES IN NOVAE AND SUPERNOVAE
}

\author{
J.M.C. RAWLINGS \\ Department of Physics \\ Nuclear Physics Laboratory \\ Keble Road, Oxford OX1 3RH, UK
}

\begin{abstract}
Molecular observations and models of the chemical processes in the ejecta of novae and supernovae are reviewed. Although only a few molecular species have been identified, the information that they give has provided great insight into the physical and chemical conditions. We now have quite a detailed understanding of the processes at work in both novae and supernovae.
\end{abstract}

\section{Introduction}

Until about ten years ago the only molecular feature seen in novae was the " $5 \mu \mathrm{m}$ excess" attributed to $\mathrm{CO} v=1 \rightarrow 0$ (or $\mathrm{C}_{3}^{+}$). Before SN1987a there was no evidence for molecule or dust formation in the ejecta of supernovae. The situation now is quite different; $\mathrm{CO}, \mathrm{CN}$, $\mathrm{SiO}, \mathrm{SiO}_{2}, \mathrm{SiC}$ and $\mathrm{PAH}$ features (as well as $\mathrm{H}_{2}$ at late times) have been seen in novae. In $\mathrm{SN} 1987 \mathrm{a} \mathrm{CO},\left(\mathrm{CO}^{+}\right), \mathrm{SiO}$ and $\mathrm{H}_{3}^{+}$have been detected. Dust has been observed in the ejecta of SN1987a and about a third of all novae produce optically thick dust shells.

Novae and supernovae are chemically and physically very dissimilar. The essential characteristics that they share are the presence of high densities (in excess of $10^{10} \mathrm{~cm}^{-3}$ ), high temperatures (2000-10000 K), harsh, intense and strongly time-dependent radiation fields and complex physical conditions (eg. the ionization structure).

\section{Novae}

\subsection{OBSERVATIONS}

Novae are of particular interest at IR wavelengths in that they are often seen to produce very optically thick dust shells (completely obscuring the nova) at a time some 10-100 days after the outburst (eg. see Ney and Hatfield 1978). They are therefore useful laboratories in which to examine the processes of dust grain nucleation and growth. Typical parameters for a classical nova are given below:

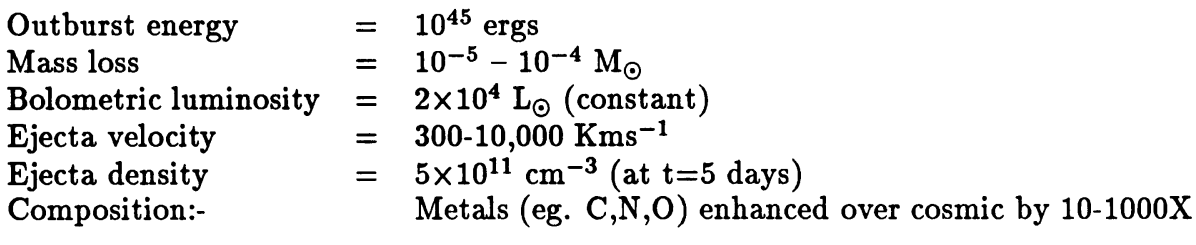


In general two physical nova types have been identified (according to the white dwarf composition):

1. C-N-O types; typically associated with duller, slow $\left(\dot{\mathrm{m}}_{v} \simeq 0^{m} \cdot 01 \mathrm{day}^{-1}\right)$, dusty novae and tend to produce an optically thick carbon dust shell (eg. FH Ser, NQ Vul). In most cases CO (at 4.8 and $2.3 \mu \mathrm{m}$ ) is the only molecule that has been detected and in the pre-dust formation epoch only. (eg. Ferland et al. 1979)

2. He-Mg-Al types; typically associated with brighter, faster novae $\left(\dot{\mathrm{m}}_{v} \simeq 0^{m} \cdot 1\right.$ day $\left.^{-1}\right)$ and tending to produce an optically thin silicate type shell if any dust is formed at all (eg. V1370 Aql, QU Vul). In these novae, the predominant molecular lines seen are of $\mathrm{SiC}, \mathrm{SiO}$ and other silicate features sitting on top of the dust continuum. (eg. Gehrz et al. 1984)

This is, however, a very general categorization (Gehrz 1990) and there are several recent novae which do not easily fit into these categories. A notable example is V842 Cen (1986) (Gehrz 1990): At least three different dust/molecular types seem to have formed in the ejecta; PAH features at 3.28 and $3.4 \mu \mathrm{m}$ were detected in the ejecta shortly after the transition. The relative strength of these features changed substantially with time which could indicate a changing structural nature (Hyland and McGregor 1988). The origin of the PAH features is likely to be the destruction of dust grains by shocks associated with the progress of the ionization fronts (Rawlings and Evans 1991). The subsequent exposure to the intense UV and ions such as $\mathrm{C}^{+}$will lead to the eventual destruction of the PAHs.

In addition, the presence of both carbon and silicate dust is required so as to account for the spectrum between 7 and $13 \mu \mathrm{m}$. A feature at $11.3 \mu \mathrm{m}$ could be due to PAHs or annealed olivine. Amorphous olivine smokes also gives very good fits to the $8-13 \mu \mathrm{m}$ spectra of several "silicate" type novae although the absence of the $20 \mu \mathrm{m}$ O-Si-O bend feature in many novae is puzzling (Roche et al. 1984).

\subsection{MODELS}

To date, only carbon-rich novae have been studied in any detail.

The chemical modelling has concentrated on two epochs of the nova evolution:

- The pre-dust formation epoch, when the CO $5 \mu \mathrm{m}$ feature is seen (Rawlings 1986,1988).

- The dust nucleation epoch (Rawlings and Williams 1989). This is the first attempt at detailed chemical modelling of the kinetics of the formation of nucleation sites.

A 'typical' nova may maintain a constant bolometric luminosity for several hundreds of days after the outburst. Thus, as the ejecta expands, the effective photosphere contracts and the radiation field hardens. As a result, the expanding ejecta is overtaken by a series of ionization fronts. In the studies of the chemistry at early times (Rawlings 1986) it was found that simple molecules such as $\mathrm{H}_{2}$ and $\mathrm{CO}$ could have appreciable abundances only in the region where the carbon is neutral (CI). In these conditions the carbon continuum $(\lambda \leq 1100 \AA)$ and the $\mathrm{H}_{2} / \mathrm{CO}$ self-mutual shielding protect the molecules from the radiation field. As a result of the intensity of the radiation field and the high densities, the chemistry is in steady-state (at $\mathrm{T} \simeq 3500 \mathrm{~K}$ ). The main $\mathrm{H}_{2}$ formation routes are by three-body and $\mathrm{H}^{-}$reactions. The main loss route is collisional dissociation by atomic hydrogen. In the CII region $\left(\mathrm{T} \simeq 6000 \mathrm{~K}\right.$ ) the $\mathrm{H}_{2}$ can not build up appreciable column densities due to the presence of the unshielded Lyman flux. In a model of the CI region limited to $\mathrm{H}, \mathrm{C}$ and $\mathrm{O}$ chemistry, the only other molecules to achieve abundances greater than about $10^{-10}$ are 
$\mathrm{CH}, \mathrm{OH}, \mathrm{C}_{2}$ and $\mathrm{O}_{2}$. The chemistry is also very temperature sensitive: temperatures of less than $3500 \mathrm{~K}$ are required for $\mathrm{H}_{2}$ to be optically thick. The $\mathrm{CO}$ formation route is typical for hot circumstellar environments:

$$
\begin{gathered}
\mathrm{O}+\mathrm{H}_{2} / \mathrm{H} \longrightarrow \mathrm{OH}, \quad \mathrm{O}^{-}+\mathrm{H}_{2} \longrightarrow \mathrm{OH} \\
\mathrm{C}+\mathrm{H}_{2} / \mathrm{H} \longrightarrow \mathrm{CH}, \quad \mathrm{C}^{-}+\mathrm{H}_{2} \longrightarrow \mathrm{CH} \\
\mathrm{OH}+\mathrm{C} \longrightarrow \mathrm{CO}+\mathrm{H}, \quad \mathrm{CH}+\mathrm{O} \longrightarrow \mathrm{CO}+\mathrm{H}, \quad \mathrm{C}+\mathrm{O} \longrightarrow \mathrm{CO}+h \nu
\end{gathered}
$$

The main $\mathrm{CO}$ loss routes are photodissociation and collisional dissociation by $\mathrm{H}$ atoms. Simple ionization models suggest that the neutral zone ceases to exist within a few days of the outburst. This is contradicted by the presence of $\mathrm{CO}$ at later times. If, however, a thin $\left(\Delta \mathrm{r} / \mathrm{r}_{e j} \simeq 0.01\right)$, cool, neutral shell of ejecta of enhanced density could survive, then the observationally deduced column density of $>10^{18} \mathrm{~cm}^{-2}$ could be sustained.

When modelling the chemical formation of dust nucleation sites we should note that the ejectae of novae are very far from LTE and a microscopic approach is required. The intensity of the radiation field together with the extreme inefficiency of molecule formation in ionized regions limits nucleation to the $\mathrm{CI}$ ionization zone (Rawlings and Williams 1989). At the temperatures and densities appropriate to the nucleation epoch, $\mathrm{X}\left(\mathrm{H}_{2}\right) \simeq 10^{-4}$ $10^{-2}$ and $\mathrm{CO}$ saturates in this region. This is an important point since $\mathrm{C}>\mathrm{O}$ in the ejecta. The only viable nucleation mechanism is based on an extended hydrocarbon chemistry. This chemistry is limited to molecules containing 8-10 carbon atoms (ring closure rapidly stabilizes molecules with greater than about 10 carbon atoms or less) and incorporates all data that is available on large molecule chemistry. The saturation of $\mathrm{CO}$ prevents oxygen attack on the hydrocarbons which would inhibit the formation of nucleation sites.

It is found that small species (such as $\mathrm{C}_{2}$ ) are more important 'building blocks' to nucleation sites than larger molecules. This is due to the radiation field which keeps the abundance of the larger molecules low. In addition the chemistry is extremely sensitive to the density, fractional ionization and $\mathrm{H}_{2}$ abundance - an ionization of less than $10^{-3}$ and $\mathrm{X}\left(\mathrm{H}_{2}\right)>10^{-4}$ are required for nucleation sites to be formed in sufficient abundance. This in turn requires the gas temperature to be low $(1000-1500 \mathrm{~K})$ and the shell density to be enhanced over the spherical mean by a factor of 50 or more.

\section{Supernovae}

\subsection{OBSERVATIONS}

SN1987a was the first supernova in which molecules and dust were seen to form. The extensive coverage has revealed the presence of several molecular species. CO $2.3 \mu \mathrm{m}$ and $4.6 \mu \mathrm{m}$ were detected at times of 112 and 117 days post-outburst onwards respectively (Meikle et al. 1989). Fitting to the $2.3 \mu \mathrm{m}$ line shows an apparent rise of the CO mass with time (from Spyromilio et al. 1988):

$\begin{array}{lllll}\frac{\mathrm{t} / \text { days }}{192} & \frac{\mathrm{T} / \mathrm{K}}{3000} & \frac{\mathrm{v}\left(\mathrm{Kms}^{-1}\right)}{2000} & \frac{\mathrm{M}_{c o} / \mathrm{M}_{\odot}}{1.7 \times 10^{-5}} \\ 255 & 1800 & 1200 & 4.7 \times 10^{-5} \\ 284 & 1600 & 1200 & 1.2 \times 10^{-4}\end{array}$

Note that the implied $\mathrm{CO}: \mathrm{C}$ ratio $\left(10^{-3}\right)$ is very high. The same authors have suggested that $\mathrm{CO}^{+}$may have been present in the ejecta at 255 days. The identification is however weak and could quite easily be explained by ArII fine structure. 
SiO $\mathrm{v}=1 \rightarrow 0$ emission at $8.1 \mu \mathrm{m}$ was first seen at 160 days and was clearly visible in the period 450-578 days post-outburst. Roche et al. (1991) have fitted the emission spectrum with an SiO temperature of $1500 \mathrm{~K}$ and a total mass of $4 \pm 2 \times 10^{-6} \mathrm{M}_{\odot}$ (corresponding to about $15 \%$ of the total dust mass).

Miller et al. (1991) have identified and fitted $\mathrm{H}_{3}^{+}$to the features at about 3.4 and 3.5 $\mu \mathrm{m}$ as seen in the day 192 spectra of Meikle et al. (1989). They find the $\mathrm{H}_{3}^{+}$mass to be $1.1 \times 10^{-7} \mathrm{M}_{\odot}$ with an excitation temperature of $2050 \mathrm{~K}$.

\subsection{MODELS}

The features of the supernova ejecta that distinguish it are that the ejecta is both chemically and physically highly stratified and the main radiation field is an intrinsic source function derived from the radioactive decay of ${ }^{56} \mathrm{Co}$.

Of the various models that have been developed so as to describe the behaviour of SN1987a, one of the most successful has been the partially mixed model 10HM of Pinto and Woosley (1988). Some characteristics of this model (at $t=1$ year) are given below:

$\begin{array}{lll} & \text { CORE } & \text { MANTLE } \\ \mathrm{v}\left(\mathrm{kms}^{-1}\right) & 400-1000 & 1500-3500 \\ \mathrm{n}\left(\mathrm{cm}^{-3}\right) & 1.1 \times 10^{9} & 6.7 \times 10^{8} \\ \mathrm{~T}(\mathrm{~K}) & 2000-3000 & 6000-7000 \\ \mathrm{X}_{H} & \leq 0.001 & 0.45 \\ \mathrm{X}_{H e} & 0.54 & 0.54\end{array}$

Note that hydrogen is highly deficient in the core. This is of great interest as the velocity and temperature of the $\mathrm{CO}$ emission lines are consistent with the core being the origin of emission. We may therefore be seeing evidence of a non-hydrogen based chemistry.

The situation is further complicated by the radiation field. The $\mathrm{He}^{\star}\left(2^{1} \mathrm{~S}\right)$ metastable state of helium is indirectly excited by the decay of ${ }^{56} \mathrm{Co}\left(\lambda^{-1} \simeq 112\right.$ days $)$. The consequent decay to the ground state results is a strongly time- and density-dependent radiation field. An estimate of the strength of this field has been made by Petuchowski et al. (1989) and Rawlings and Williams (1990) on the basis of Fe ionization ratios. In addition there are contributions from the photospheric radiation field (of constant temperature $5500 \mathrm{~K}$ ) and the decay of ${ }^{57} \mathrm{Co}\left(\lambda^{-1} \simeq 392\right.$ days $)$. Some $80 \%$ of all the fast electron energy is deposited in the core region (McCray 1990).

In addition to the radiation field, the fast electron flux has direct consequences on the chemistry; collisional ionizations lead to a high $\mathrm{He}^{+}$abundance which then attacks any molecular species (such as $\mathrm{CO}$ ) which may be present. Collisional detachments of negative ions are also significant.

In these conditions the most effective molecular formation routes will be simple one-step reactions such as the radiative association:

$$
\mathrm{C}+\mathrm{O} \longrightarrow \mathrm{CO}+\mathrm{h \nu}
$$

The rate for this reaction has recently been calculated by Dalgarno et al. (1989). Other important reaction types are destruction by $\mathrm{He}^{+},\left(\mathrm{CO}+\mathrm{He}^{+} \longrightarrow \mathrm{He}+\mathrm{C}^{+}+\mathrm{O}\right)$ and photons, negative ion reactions, charge exchanges, three-body reactions (with $\mathrm{He}$ as the third body), dissociative recombinations and collisional dissociations.

Petuchowski et al. 1989 [1], Rawlings and Williams 1990 [2], and Lepp et al. 1990) [3], have developed models of the chemistry in which it was found that the $\mathrm{CO}$ formation occurs via several channels. The direct radiative association referred to above is dominant 
but formation via $\mathrm{C}^{-}, \mathrm{C}_{2}$, and $\mathrm{O}_{2}$ are all significant. The $\mathrm{CO}$ formation rate is therefore robust to parameter changes and the $\mathrm{CO}$ abundance is essentially controlled by the efficacy of the destruction mechanisms (photoionization/dissociation and reaction with $\mathrm{He}^{+}$). The models differ in the relative importance that they ascribe to $\mathrm{CO}$ destruction by the radiation field and $\mathrm{He}^{+}$. Different hydrogen abundances are also assumed (hydrogen chemistry is only significant in the model of [3]). A much greater $\mathrm{CO}^{+}$abundance is predicted by the model of [3] than those of [1] or [2] but both [2] and [3] predict $\mathrm{CO}$ abundances that are $100 \mathrm{X}$ too small as compared to the observations. Lepp et al. [2] point out that this could be improved if the charge exchanges of $\mathrm{He}^{+}$with low IP metals have rate coefficients of $3.3 \times 10^{-9} \mathrm{~cm}^{3} \mathrm{~s}^{-1}$. What is more probable is that the supernova ejecta is poorly mixed. Rayleigh-Taylor instabilities may then form at the boundaries of the abundance discontinuities so that fingers of mantle material are pushed back into the core region. The CO emission could then originate from this low velocity mantle material. If this is the case then both models predict a $\mathrm{CO}$ mass that could rise to as high as $10^{-2} \mathrm{M}_{\odot}$ at late times. The only other molecular species predicted to be present are $\mathrm{SiO}, \mathrm{C}_{2}, \mathrm{O}_{2}, \mathrm{H}_{3}^{+}$and $\mathrm{HeH}^{+}$.

\section{The Future}

The direct associative ionization reaction of $\mathrm{H}(\mathrm{n}=2)$ with $\mathrm{H}$ to form $\mathrm{H}_{2}^{+}$has been shown to be highly significant in dense protostellar outflows (Rawlings et al. 1991). Miller et al. (1991) have suggested that (as a result of the reaction of $\mathrm{H}_{2}^{+}$with $\mathrm{H}_{2}$ ) this may be the main formation route of $\mathrm{H}_{3}^{+}$in $\mathrm{SN} 1987 \mathrm{a}$. It is likely that reactions involving $\mathrm{H}(\mathrm{n}=3)$ may be even more important. In any case, as data on these newly studied reaction types emerges we can expect major alterations in our understanding of the chemistry in these environments.

\section{References}

Dalgarno, A., Du, M.L. and You, J.H. 1990, Astrophys. J., 349675

Ferland, G.J., et al. 1979, Astrophys. J., 227489

Gehrz, R.D., et al. 1984, Astrophys. J., 281303

Gehrz, R.D. 1990, In: "Physics of Classical Novae" (Springer-Verlag), Eds. A. Cassatella and $R$. Viotti

Hyland, A.R. and McGregor, P.J. 1988, In "Interstellar Dust", IAU Symposium No. 135

Lepp, S., Dalgarno, A. and McCray, R. 1990, Astrophys. J., 358262

McCray, R. 1990, In: "Molecular Astrophysics" (CUP), Ed. T. Hartquist

Meikle, W.P.S., et al. 1989, Mon. Not. R. ast. Soc., 238193

Miller, S., Tennyson, J., Lepp, S. and Dalgarno, A. 1991 Submitted to Nature

Ney, E.P. and Hatfield, B.F. 1978, Astrophys. J., 219 L111

Petuchowski, S.J., Dwek, E., Allen, J.E. Jr. and Nuth, J.A. 1989, Astrophys. J., 342406

Pinto, P.E. and Woosley, S.F. 1988, Astrophys. J., 329820

Rawlings, J.M.C. 1986, Ph.D. Thesis, UMIST

Rawlings, J.M.C. 1988, Mon. Not. R. ast. Soc., 232507

Rawlings, J.M.C. and Williams, D.A. 1989, Mon. Not. R. ast. Soc., 240729

Rawlings, J.M.C. and Williams, D.A. 1990, Mon. Not. R. ast. Soc., 246208

Rawlings, J.M.C., Drew, J.E. and Barlow, M.J. 1991, These proceedings

Rawlings, J.M.C. and Evans, A. 1991, In preparation

Roche, P.F., Aitken, D.K. and Whitmore, B. 1984, Mon. Not. R. ast. Soc., 211535

Roche, P.F., Aitken, D.K. and Smith, C.H. 1991, Mon. Not. R. ast. Soc., 252 39P

Spyromilio, J., Meikle, W.P.S., Learner, R.C.M. and Allen, D.A. 1988, Nature, 334327 


\section{QUESTIONS AND ANSWERS}

V.Escalante: At which stage of the nova outburst did you carry out the chemistry calculations? We know that red giant stars produce dust. How do we know that the dust observed in a nova outburst comes from the outburst itself and not from the red giant companion?

J.M.C.Rawlings: Calculations are performed at times that are consistent with observations. Thus the pre-dust formation chemistry is studied over the period $3-20$ days post-outburst (when CO is seen) and the dust nucleation chemistry is studied from $\sim 30$ to 80 days post-outburst (typically 50 days) ie. immediately prior to dust formation. There are many reasons why the dust cannot originate from the red star. I list a few below: (i) dust formation can result in an optically thick dust shell which completely covers the nova 'sky'. Dust picked up from the red star would be localized into one area; (ii) the radius of the IR pseudo-photo sphere and the dust temperature are consistent with rapid condensation - in any case the blackbody radius of the dust shell is very much larger than the binary separation; (iii) it is highly unlikely that any pre-existent dust will survive a wind moving at between 500 and $10,000 \mathrm{~km} / \mathrm{s}$.

J.P.Maillard: Providing the detection of $\mathrm{H}_{8}^{+}$is correct - which is not completely convincing at the resolution of the spectrum - what would be the rate of production of the $\mathrm{HeH}+$ ? And did you look at it because the fundamental band of $\mathrm{HeH}^{+}$is located in the same spectral range 3 to $4 \mu \mathrm{m}$ ?

J.M.C.Rawlings: This work was done by Miller et al. As we have two of the authors here I will pass this question to one of them:

S.Lepp: Our models produce between 10 and 100 times less $\mathrm{HeH}^{+}$than $\mathrm{H}_{3}^{+}$. Still we have tentatively identified two lines as being from vibrationaly excited $\mathrm{HeH}^{+}$.

J.A.de Freitas Pacheco: Concerning your dust calculations for novae, what CNO enhancement have you assumed?

J.M.C.Rawlings: We have considered various enhancements in the range 10 times to 100 times (being compatible with the observations). In the results that I have presented here we assume enhancement of about 50 times. Note that, of course, we require $C>O$ in this model.

M.Guelin: Did anybody detect $C^{18} O$ in the ejecta of SN1987A? Would it be possible to derive the $C^{18} \mathrm{O} / \mathrm{C}^{16} \mathrm{O}$ abundance ratio? ${ }^{18} \mathrm{O}$ could be comparable to ${ }^{16} \mathrm{O}$ in these ejecta?

J.M.C.Rawlings: To my knowledge $C^{18} O$ has not been detected in SN1987a, but I would not like to make a definitive statement on that. The problem is that the near infra-red spectrum is heavily erowded with Ar II and Nickel lines. I would also think it unlikely that an abundance ratio could be determined as the CO emission is almost certainly very optically thick.

S.Lepp: I just wanted to comment that in a recent work by Liu, Dalgarno and Lepp, we have analysed the CO spectra and found that much larger CO masses may be fit, when optical depth and non-LTE populations are accounted for.

J.M.C.Rawlings: Yes, I think our models have predicted this to be in the core: either conditions are unfavorable in which case $C O$ has a very low abundance or, in favorable situation $C O$ is very optically thick. The condition required so as to predict the $\mathrm{CO}$ mass deduced in the optically thin approximation would have to be somewhat contrived. 